\title{
Development of a scanning surface probe for nanoscale tip-enhanced desorption/ablation
}

\author{
Kent A. Meyer, ${ }^{1}$ Olga Ovchinnikova, ${ }^{1}$ Kin $\mathrm{Ng}^{2}$ and Douglas E. Goeringer ${ }^{1, \mathrm{a})}$ \\ ${ }^{1}$ Organic and Biological Mass Spectrometry Group, Chemical Sciences Division, \\ Oak Ridge National Laboratory, Oak Ridge, Tennessee 37831-6131, USA \\ ${ }^{2}$ Department of Chemistry, California State University-Fresno, 2555 E. San Ramon, Fresno, \\ California 93740-8034, USA
}

(Received 23 September 2008; accepted 27 November 2008; published online 23 December 2008)

\begin{abstract}
We report on the development of a versatile scanning apparatus for nanoscale surface sampling that utilizes the interaction of laser radiation at a sharp probe tip to effect desorption/ablation on opaque substrates. The process, which currently yields surface craters as small as $\sim 50 \mathrm{~nm}$ diameter $\times 5 \mathrm{~nm}$ deep, has been demonstrated with both metal-coated and bare silicon tips. Desorption/ ablation under the tip occurs at illumination intensities below the corresponding optical far-field threshold, suggesting that the latter process should not degrade the spatial resolution attainable for proposed chemical imaging methods based on the scanning surface probe. () 2008 American Institute of Physics. [DOI: 10.1063/1.3053200]
\end{abstract}

\section{INTRODUCTION}

Scanning probe microscopy (SPM) techniques, such as scanning tunneling microscopy ${ }^{1}$ and atomic force microscopy $(\mathrm{AFM}){ }^{2}$ are versatile surface imaging methods that provide the capability for ascertaining the structural framework of matter on the atomic scale. Such instruments use various interaction forces between a sharp, nanometer-scale probe tip to monitor and control the tip-to-sample distance. Correlating the control signal with the tip location as it is scanned across a surface then enables nanoscale topographic images to be acquired for groups of individual atoms and molecules. Moreover, when laser light is focused onto the probe, ${ }^{3,4}$ its acts as an optical antenna producing strong enhancement of the radiation intensity in a localized region at the apex and comparable with the radius of curvature of the probe tip (i.e., near-field enhancement). Metal tips can locally enhance electric fields up to $10^{3}$ if the wavelength of light is in resonance with surface plasmons. ${ }^{5}$ Thus, the probe can function as a confined, intense, near-field source, the resolution essentially being defined by the tip diameter. By subsequently collecting the scattered light in the far field, it is possible to image features below the diffraction limit; the final instrumental configuration can take a number of forms but is known collectively as apertureless near-field scanning optical microscopy (NSOM). Furthermore, because this effect results in orders-of-magnitude signal gain, metal-probe imaging has been combined with fluorescence, ${ }^{6}$ Raman, ${ }^{7}$ and infrared $^{8}$ spectroscopies to yield additional chemical information at the nanometer scale.

The enhanced radiation intensity responsible for NSOM optical spectroscopy also can produce nanoscale surface effects, so that the tip can serve as a desorption/ionization source for mass spectrometry (MS) as well. Since MS can

\footnotetext{
a) Author to whom correspondence should be addressed. Electronic mail: goeringerde@ornl.gov.
}

yield substantial molecular information in the form of molecular mass and detailed structural characterization and can be combined with atmospheric pressure ionization sources, tip-enhanced desorption/ablation MS surface imaging has enormous potential as an alternate spectroscopic method for investigating nanoscale structures. Nanoscale MS imaging has a rich history which started with work involving secondary ionization methods. ${ }^{9}$ Laser-desorption based nanoscale MS imaging is itself over 10 years old, yet only a few methods have been reported and optimal instrumental designs for it are still under investigation. For example, micrometer to submicrometer lateral resolution MS analysis has been achieved in a few instances by using a near-field source and extracting the desorbed species into a time of flight MS..$^{10,11}$ However, in neither case was ionization performed at atmospheric pressure, was an apertureless probe used, nor was topographical information acquired to produce chemical images. Other novel designs have nanoscale sensitivity but have not been reported to form a full image with nanometersized pixels. ${ }^{12}$

Consequently, we are pursuing development of an instrument for atmospheric pressure, nanoscale topographic, and chemical imaging of surfaces via MS. Below we describe the initial task in the overall development process, construction, and operation of a versatile scanning surface probe for nanoscale tip-enhanced laser desorption/ablation. In addition, we relate its use for experimental investigations of absorption of near-field radiation as the mechanism for the desorption/ ablation process, since other effects such as tip heating, deformation (nanoindentation) due to tip expansion, secondary particle bombardment, etc., also could be responsible.

\section{INSTRUMENTATION}

A rendered drawing of the near-field nanoscale instrument used in our investigations is shown in Fig. 1. A Veeco Metrology (Santa Barbara, CA) MultiMode AFM head and 


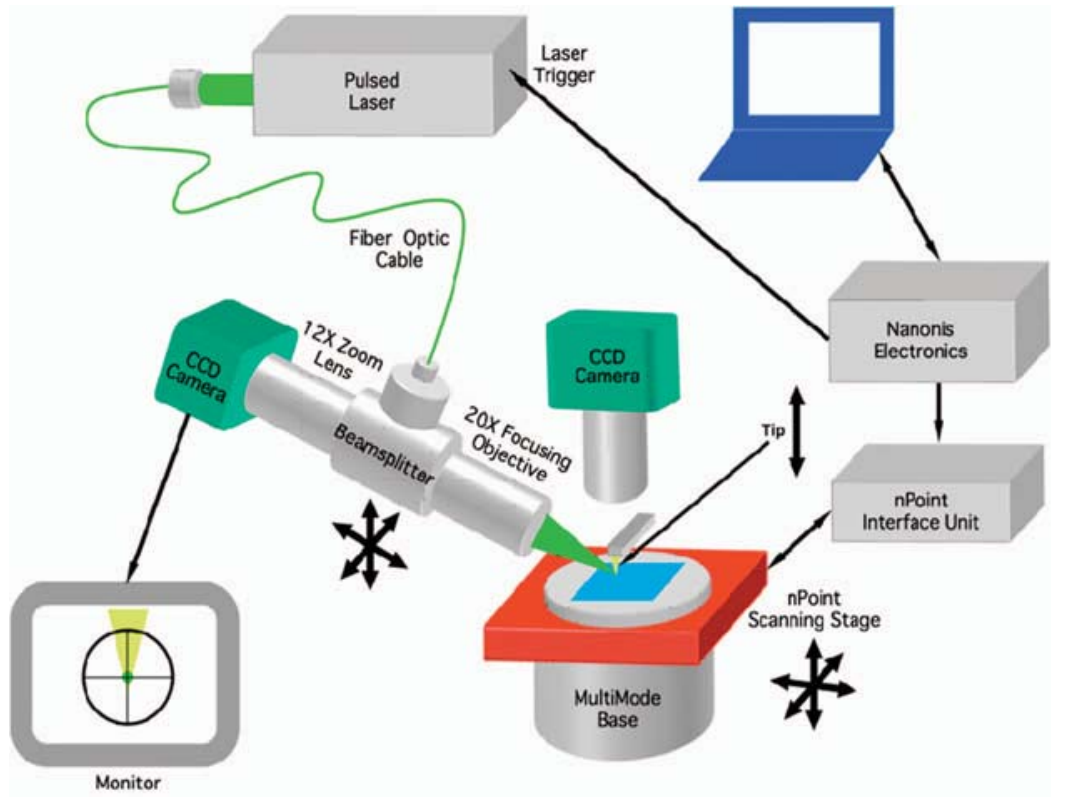

FIG. 1. (Color) Sketch of tip-enhanced nanoscale laser desorption apparatus designed and used in these studies.
nPoint (Madison, WI) sample stage (model iCXYZ100-DM: $15 \mu \mathrm{m} z ; 100 \mu \mathrm{m} x, y$ ranges) comprise the tip/sample mechanical subsystem. Cantilever-mounted AFM probes are placed in a holder that, when inserted into the AFM head, positions the cantilever at $\sim 12^{\circ}$ with respect to the sample surface. A low-power cw diode laser beam, reflecting off the back face of the cantilever, provides topographical information through its deflection as the tip moves across the surface. The nPoint stage is powered by a digital closed-loop controller (C-300) that enables nanometer-scale $x, y$ positioning/scanning of the sample with little drift and hysteresis over the course of an experiment, thereby allowing for array-based sampling protocols over relatively long time periods. The probes used here are standard cantilever-mounted silicon AFM tips with conical tapers, having ultimately nanometer-sized tip curvature and different compositions, such as metal coatings or dopant changes.

A telescope-based optical subsystem, situated approximately orthogonal to the long axis of the cantilever, transfers laser radiation to the tip and underlying sample surface. Geometrical constraints imposed by protrusion of the cantilever edge into the optical path between the tip apex and laser beam restrict the associated minimum polar angle to $\sim 60^{\circ}$, which is used here. Laser pulses from a frequency-doubled Minilite (Continuum, Santa Clara, CA) neodymium doped yttrium aluminum garnet (Nd:YAG) laser $(532 \mathrm{~nm}, \sim 5 \mathrm{~ns}$ pulse width), running in single-shot mode, enter a mechanically stabilized multi- or single-mode fiber and become collimated via an anti-reflection-coated FiberPort (OFR, Caldwell, NJ) on exiting into the side arm of the alignment system. After the pulses pass through an adjustable linear polarizer and reflect toward the sample via a nonpolarizing cube beamsplitter, they are focused using a Mitutoyo M Plan Apo SL $20 \times$ microscope objective; the result is a far-field elliptical spot at the surface $(\sim 50 \times 100 \mu \mathrm{m}$ axes when using the multimode fiber). The linear polarizer enables the polarization of the laser radiation to be adjusted either parallel or perpendicular to the tip cone center axis.
The same optical subsystem used for laser beam steering and focusing is also employed for laser focus/tip alignment and sample observation. The image of the tip/sample region is sent back into the objective, from which it then transits the cube beamsplitter before passing through a $12 \times$ zoom lens, a second adjustable linear polarizer, a $650 \mathrm{~nm}$ cutoff optical filter, a reticle, and finally focusing on a charge-coupled device (CCD) continuous imaging camera. The second polarizer prevents secondary laser reflections off the cube beamsplitter from overwhelming and/or damaging the CCD camera, and the filter blocks the AFM laser diode light. The reticle acts a convenient sight for subsequent positioning of the tip and laser focus once they are initially aligned via remote-controlled Picomotor ${ }^{\mathrm{TM}}$ (New Focus, San Jose, CA) actuators. The tip/sample region can also be viewed from another perspective (i.e., normal to the sample surface) via a second $12 \times$ zoom lens/CCD camera setup located directly over a window in the top of the MultiMode head. White light illumination of the tip/sample region is performed using incandescent/fluorescent lighting.

AFM control and data acquisition are performed using the Nanonis (Zurich, Switzerland) SPM software and controller, which is interfaced to the MultiMode via the nPoint C-300. The Nanonis programming interface is employed to create custom LABVIEW codes that control the $x, y, z$ positioning of the tip relative to the surface as well as the triggering of laser pulses.

\section{RESULTS AND DISCUSSION}

Tip-enhanced laser desorption/ablation experiments were carried out via the following sequence of events: the tip, after being brought into contact with the surface, was moved lightly across the sample to a given location and was then raised off the surface by a specified amount; a single laser pulse focused at the tip/surface region was triggered immediately thereafter; last, the tip was withdrawn to a relatively high distance $(\sim 5 \mu \mathrm{m})$. Topographical imaging of the 

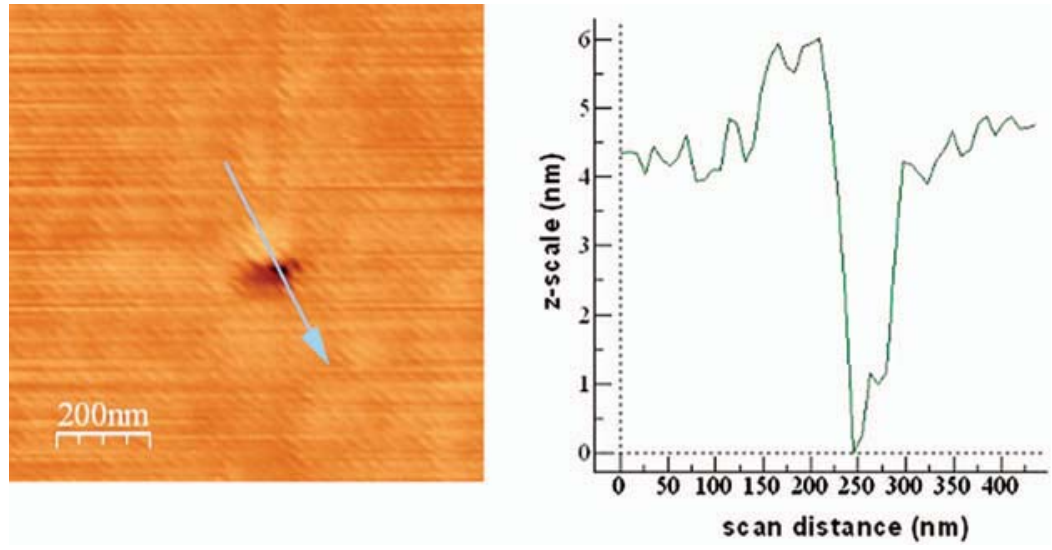

FIG. 2. (Color) AFM image of a laser desorbed nanohole on Sharpie ${ }^{\circledR}$ Red surface using an unmodified MikroMasch NSC15 silicon tip. The tip was located $3 \mathrm{~nm}$ above the surface before the laser pulse was applied. The laser pulse was polarized linearly along the axis of the tip and was applied in a direction from bottom to top in the image. The blue line and arrow indicates the line and direction (left to right) at which the profile at right was recorded. The raised feature in the image was not present in the blank and possibly indicates where ablated material condensed under these conditions. Images were created by WSXM 4.0 software (Ref. 19).

region in the vicinity of the affected area was subsequently performed using the AFM's standard tapping mode. Blank measurements, which are performed using the above procedure without laser triggering, were often used to determine if mechanical damage was possible with the experimental conditions.

For a model analyte, Sharpie® Red marker, known to contain the dye Rhodamine B, was swiped onto glass cover slips. A deep red surface deposit, believed to be mostly a dye layer tens of nanometers or greater thick, remained after vacuum drying over several hours. Blank AFM scans revealed 25-100 $\mu \mathrm{m}^{2}$ regions of relative smoothness (e.g., $\pm 0.5 \mathrm{~nm})$ in which the experiments described below were carried out.

Figure 2 shows an AFM image of the affected sample region following tip-enhanced ablation using an uncoated NSC15 tip (MikroMasch, San Jose, CA) and a single $15 \mathrm{~nJ}$ laser pulse, with other conditions indicated. The crater profile indicates a full width at half maximum of $\sim 50 \mathrm{~nm}$ with a depth of $\sim 4-5 \mathrm{~nm}$. Laser far-field damage was not observed for $15 \mathrm{~nJ}$ laser pulses when the tip was retracted. By gradually increasing the laser energy with the same far-field focusing conditions, the corresponding damage threshold was estimated to be $\sim 1 \mu \mathrm{J} /$ pulse $\left(\sim 25 \mathrm{~mJ} / \mathrm{cm}^{2}\right)$, suggesting that the tip enhancement for desorption is $\sim 100$ times or greater. For reproducibility assessment of tip-enhanced ablation, large-scale arrays of $>200$ similar ablation craters, each resulting from a single $\sim 15 \mathrm{~nJ}$ laser pulse, were also analyzed via AFM imaging. Craters were observed throughout the entire array, allowing us to conclude high reproducibility and enabling us to perform statistical analyses of hole depths that were independent of tip degradation. Examination of a later series of craters created under similar conditions showed a variation of $\sim 50 \%$ in depth. It is yet unclear as to whether shot-to-shot laser fluctuations, laser "hot spots" from multimode fiber focusing, the surface and/or environment, precision in tip placement above the surface, or other factors are responsible for the variability. ${ }^{13}$

Polarization studies performed using the NSC15 tip were not able to discern whether the holes created were strictly due to electric-field enhancement. Furthermore, and contrary to expectation for a near-field tip enhancement mechanism, nanoholes were generated with the NSC15 tip positioned as high as $\sim 400 \mathrm{~nm}$ above the surface. Field enhancement should become insignificant when the tip-sample distance is greater than $30 \mathrm{~nm} .^{14,15}$ It is possible that both thermal and electric-field induced effects can be responsible for the holes that we observed. However, according to the temperature profile simulations performed by Downes et al., ${ }^{12}$ no substantial temperature jump should be induced in an unmodified Si tip when using laser peak powers similar to those in our experiments. Zeisel et al. ${ }^{16}$ argued that a ballistic heat transfer mechanism, in which ablation occurs via surface bombardment by atoms ejected through transient tip heating, was likely responsible for formation of nanoscale holes on the surface of an anthracene crystal exposed to pulsed laser radiation delivered through metallized fiber tips. In the case of Rhodamine (an ionic solid), however, workers ${ }^{17}$ in the same laboratory never observed nanoablation by ballistic heat transfer, presumably because its heat of sublimation is significantly higher than that for anthracene (a van der Waals solid). A fourth possible effect, that of mechanical indentation due to inadvertently forcing the tip against the test surface during experiments, was scrupulously tested for and was determined not to be a factor. The observations suggest that other possible effects than the four above may be important to consider in future work.

In addition to Rhodamine $\mathrm{B}$, we have investigated indium tin oxide (ITO) coated glass coverslips. Gold-coated tips were utilized to effect nanoscale-sized (e.g., $\sim 150 \mathrm{~nm}$ diameter $\times 3 \mathrm{~nm}$ deep) ablation at energies below the ITO far-field damage threshold (roughly $3 \mathrm{~J} / \mathrm{cm}^{2}$ with our laser setup). These results, taken with those of the Rhodamine surface, indicate that a wider variety of samples and substrates can be investigated. Different laser wavelengths [e.g., the third-harmonic $(355 \mathrm{~nm})$ of $\mathrm{Nd}: Y A G]$ can also be used in the future, although it is important to note that some tip enhancement factors may be dependent on the excitation wavelength. ${ }^{18}$

\section{CONCLUSIONS}

We have developed a versatile scanning apparatus for surface sampling that utilizes the interaction of laser radiation at a sharp probe tip to effect nanoscale desorption/ ablation on opaque substrates. Our experimental results also show nanoscale ablation at laser intensities below the corresponding far-field ablation threshold. Consequently, we be- 
lieve that the latter process should, upon further optimization, not degrade the spatial resolution attainable for proposed chemical imaging methods based on the scanning surface probe. Furthermore, because electric fields can be highly concentrated at the laser-illuminated tip, the potential capability to isolate associated higher-order interactions there may enable the observation and understanding of effects not seen or distinguished in far-field experiments. We expect that analysis of nanoablation events via MS and other high sensitivity techniques will be an important means of resolving the underlying mechanisms responsible for our current observations.

\section{ACKNOWLEDGMENTS}

We thank S. Jesse and S.V. Kalinin for instrument loans and their helpful suggestions in regard to selection of key pieces of equipment for the project. W. B. Whitten and R. W. Shaw are acknowledged for valuable discussions in connection with experiments. G. J. Van Berkel is recognized for his continued support and encouragement during the course of the project. Research sponsored by the Laboratory Directed Research and Development Program of Oak Ridge National Laboratory, managed by UT-Battelle, LLC, for the U. S. Department of Energy.
${ }^{1}$ G. Binning, H. Rohrer, C. Gerber, and E. Weibel, Phys. Rev. Lett. 49, 57 (1982).

${ }^{2}$ G. Binnig, C. F. Quate, and C. Gerber, Phys. Rev. Lett. 56, 930 (1986).

${ }^{3}$ Y. Inouye and S. Kawata, Opt. Lett. 19, 159 (1994).

${ }^{4}$ F. Zenhausern, M. P. Oboyle, and H. K. Wickramasinghe, Appl. Phys. Lett. 65, 1623 (1994).

${ }^{5}$ A. Downes, D. Salter, and A. Elfick, J. Phys. Chem. B 110, 6692 (2006).

${ }^{6}$ E. J. Sanchez, L. Novotny, and X. S. Xie, Phys. Rev. Lett. 82, 4014 (1999).

${ }^{7}$ A. Hartschuh, N. Anderson, and L. Novotny, J. Microsc. 210, 234 (2003).

${ }^{8}$ B. Knoll and F. Keilmann, Nature (London) 399, 134 (1999).

${ }^{9}$ R. Levisetti, J. M. Chabala, and Y. L. Wang, Ultramicroscopy 24, 97 (1988).

${ }^{10}$ D. A. Kossakovski, S. D. O'Connor, M. Widmer, J. D. Baldeschwieler, and J. L. Beauchamp, Ultramicroscopy 71, 111 (1998).

${ }^{11}$ R. Stockle, P. Setz, V. Deckert, T. Lippert, A. Wokaun, and R. Zenobi, Anal. Chem. 73, 1399 (2001).

${ }^{12}$ J. S. Becker, A. Gorbunoff, M. Zoriy, A. Izmer, and M. Kayser, J. Anal. At. Spectrom. 21, 19 (2006).

${ }^{13}$ A. Downes, D. Salter, and A. Elfick, Opt. Express 14, 5216 (2006).

${ }^{14}$ C. C. Neacsu, J. Dreyer, N. Behr, and M. B. Raschke, Phys. Rev. B 73, 193406 (2006)

${ }^{15}$ W. H. Zhang, B. S. Yeo, T. Schmid, and R. Zenobi, J. Phys. Chem. C 111, 1733 (2007).

${ }^{16}$ D. Zeisel, S. Nettesheim, B. Dutoit, and R. Zenobi, Appl. Phys. Lett. 68, 2491 (1996).

${ }^{17}$ B. Dutoit, D. Zeisel, V. Deckert, and R. Zenobi, J. Phys. Chem. B 101, 6955 (1997).

${ }^{18}$ Y. C. Martin, H. F. Hamann, and H. K. Wickramasinghe, J. Appl. Phys. 89, 5774 (2001).

${ }^{19}$ I. Horcas, R. Fernández, J. M. Gómez-Rodriguez, J. Colchero, J. GómezHerrero, and A. M. Baro, Rev. Sci. Instrum. 78, 013705 (2007)]. 